\title{
Complexity of the min-max (regret) versions of cut problems
}

\author{
Hassene Aissi, Cristina Bazgan, and Daniel Vanderpooten \\ LAMSADE, Université Paris-Dauphine, France \\ \{aissi, bazgan, vdp\}@lamsade.dauphine.fr
}

\begin{abstract}
This paper investigates the complexity of the min-max and min-max regret versions of the $s-t$ min cut and min cut problems. Even if the underlying problems are closely related and both polynomial, we show that the complexity of their min-max and min-max regret versions, for a constant number of scenarios, are quite contrasted since they are respectively strongly $N P$-hard and polynomial. Thus, we exhibit the first polynomial problem, $s-t$ min cut, whose min-max (regret) versions are strongly $N P$-hard. Also, min cut is one of the few polynomial problems whose min-max (regret) versions remain polynomial. However, these versions become strongly $N P$-hard for a non constant number of scenarios. In the interval data case, min-max versions are trivially polynomial. Moreover, for min-max regret versions, we obtain the same contrasted result as for a constant number of scenarios: min-max regret $s-t$ cut is strongly $N P$-hard whereas min-max regret cut is polynomial.
\end{abstract}

Keywords: min-max, min-max regret, complexity, min cut, $s-t$ min cut.

\section{Introduction}

The definition of an instance of a combinatorial optimization problem requires to specify parameters, in particular objective function coefficients, which may be uncertain or imprecise. Uncertainty/imprecision can be structured through the concept of scenario which corresponds to an assignment of plausible values to model parameters. Each scenario $s$ can be represented as a vector in $\mathbb{R}^{m}$ where $m$ is the number of relevant numerical parameters. Kouvelis and $\mathrm{Yu}[7]$ proposed the min-max and min-max regret criteria, stemming from decision theory, to construct solutions hedging against parameters variations. In minmax optimization, the aim is to find a solution having the best worst case value across all scenarios. In min-max regret problem, it is required to find a feasible solution minimizing the maximum deviation, over all possible scenarios, of the value of the solution from the optimal value of the corresponding scenario. Two natural ways of describing the set of all possible scenarios $S$ have been considered

\footnotetext{
* This work has been partially funded by grant CNRS/CGRI-FNRS number 18227 . The second author was partially supported by the ACI Sécurité Informatique grantTADORNE project 2004 .
} 
in the literature. In the discrete scenario case, $S$ is described explicitly by the list of all vectors $s \in S$. In this case, we distinguish situations where the number of scenarios is constant from those where the number of scenarios is non constant. In the interval data case, each numerical parameter can take any value between a lower and upper bound, independently of the values of the other parameters. Thus, in this case, $S$ is the cartesian product of the intervals of uncertainty for the parameters.

Complexity of the min-max (regret) versions has been studied extensively during the last decade. In the discrete scenario case, this complexity was investigated for several combinatorial optimization problems in [7]. In general, these versions are shown to be harder than the classical versions. For a constant number of scenarios, pseudo-polynomial algorithms, based on dynamic programming, are given in [7] for the min-max (regret) versions of shortest path, knapsack and minimum spanning tree for grid graphs. The latter result is extended to general graphs in [1]. However, up to now, no polynomial problem was known to have min-max (regret) versions which are strongly $N P$-hard. When the number of scenarios is not constant, these versions usually become strongly $N P$-hard, even if the underlying problem is polynomial. In the interval data case, extensive research has been devoted for studying the complexity of min-max regret versions of various optimization problems including shortest path [5], minimum spanning tree $[4,5]$ and assignment $[2]$.

We investigate in this paper the complexity of min-max (regret) versions of two closely related polynomial problems, min cut and $s-t$ min cut. Quite interestingly, for a constant number of scenarios, the complexity status of these problems is widely contrasted. More precisely, min-max (regret) versions of min cut are polynomial whereas min-max (regret) versions of $s-t$ min cut are strongly $N P$-hard even for two scenarios. We also prove that for a non constant number of scenarios, min-max (regret) min cut become strongly $N P$-hard.

In the interval data case, min-max versions are trivially polynomial. Moreover, for min-max regret versions, we obtain the same contrasted result as for a constant number of scenarios: min-max regret $s-t$ cut is strongly $N P$-hard whereas min-max regret cut is polynomial.

After presenting preliminary concepts (Section 2), we investigate the complexity of min-max (regret) versions of min cut and $s-t$ min cut in the discrete scenario case (Section 3), and in the interval data case (Section 4).

\section{Preliminaries}

Let us consider an instance of a $0-1$ minimization problem $Q$ with a linear objective function defined as:

$$
\left\{\begin{array}{l}
\min \sum_{i=1}^{m} c_{i} x_{i} \\
x \in X \subset\{0,1\}^{m}
\end{array} \quad c_{i} \in \mathbb{N}\right.
$$

This class encompasses a large variety of classical combinatorial problems, some of which are polynomial-time solvable (shortest path problem, minimum spanning tree, $\ldots$ ) and others are $N P$-difficult (knapsack, set covering, ...). 
In the discrete scenario case, the min-max (regret) version associated to $Q$ has as input a finite set of scenarios $S$ where each scenario $s \in S$ is represented by a vector $\left(c_{1}^{s}, \ldots, c_{m}^{s}\right)$. In the interval data case, each coefficient $c_{i}$ can take any value in the interval $\left[\underline{c}_{i}, \bar{c}_{i}\right]$. In this case, the scenario set $S$ is the cartesian product of the intervals $\left[\underline{c}_{i}, \bar{c}_{i}\right], i=1, \ldots, m$.

We denote by $\operatorname{val}(x, s)=\sum_{i=1}^{m} c_{i}^{s} x_{i}$ the value of solution $x \in X$ under scenario $s \in S$, by $x_{s}^{*}$ an optimal solution under scenario $s$, and by $v a l_{s}^{*}=$ $\operatorname{val}\left(x_{s}^{*}, s\right)$ the optimal value under the scenario $s$.

The min-max optimization problem corresponding to $Q$, denoted by MiNMAx Q, consists of finding a solution $x$ having the best worst case value across all scenarios, which can be stated as:

$$
\min _{x \in X} \max _{s \in S} \operatorname{val}(x, s)
$$

This version is denoted by Discrete Min-Max Q in the discrete scenario case, and by Interval Min-Max $Q$ in the interval data case.

Given a solution $x \in X$, its regret, $R(x, s)$, under scenario $s \in S$ is defined as $R(x, s)=\operatorname{val}(x, s)-v a l_{s}^{*}$. The maximum regret $R_{\max }(x)$ of solution $x$ is then defined as $R_{\max }(x)=\max _{s \in S} R(x, s)$.

The min-max regret optimization problem corresponding to $Q$, denoted by Min-MAX REGRET Q, consists of finding a solution $x$ minimizing the maximum regret $R_{\max }(x)$ which can be stated as:

$$
\min _{x \in X} R_{\max }(x)=\min _{x \in X} \max _{s \in S}\left\{\operatorname{val}(x, s)-\operatorname{val}_{s}^{*}\right\}
$$

This version is denoted by Discrete Min-Max Regret $Q$ in the discrete scenario case, and by InTERval Min-MAX REgRet $Q$ in the interval data case.

In the interval data case, for a solution $x \in X$, we denote by $c^{-}(x)$ the worst scenario associated to $x$, where $c_{i}^{-}(x)=\bar{c}_{i}$ if $x_{i}=1$ and $c_{i}^{-}(x)=\underline{c}_{i}$ if $x_{i}=0$, $i=1, \ldots, m$. Then we can establish easily that $R_{\max }(x)=R\left(x, c^{-}(x)\right)$, as shown e.g. in [9] in the specific context of the minimum spanning tree problem.

In this paper, we focus on the min-max (regret) versions of the two following cut problems:

\section{Min Cut}

Input: A connected graph $G=(V, E)$ with weight $w_{i j}$ associated with each edge $(i, j) \in E$.

Output: A cut in $G$, that is a partition of $V$ into two sets, of minimum value.

\section{$s-t$ Min CUT}

Input: A connected graph $G=(V, E)$ with weight $w_{i j}$ associated with each edge $(i, j) \in E$, and two specified vertices $s, t \in V$.

Output: An $s-t$ cut in $G$, that is a partition of $V$ into two sets $V_{1}$ and $V_{2}$, with $s \in V_{1}$ and $t \in V_{2}$, of minimum value.

In order to prove our complexity results we use the two following problems proved strongly $N P$-hard in $[6]$. 


\section{Min Bisection}

Input: A graph $G=(V, E)$ with an even number of vertices.

Output: A bisection in $G$, that is a partition of $V$ into two equal cardinality sets, of minimum value.

$s-t$ Min Bisection

Input: A graph $G=(V, E)$ with an even number of vertices, and two specified vertices $s, t \in V$.

Output: An $s-t$ bisection in $G$, that is a partition of $V=V_{1} \cup V_{2}$ such that $s \in V_{1}, t \in V_{2}$, and $\left|V_{1}\right|=\left|V_{2}\right|$, of minimum value.

\section{Discrete scenarios case}

We show in this section the first polynomial-time solvable problem, $s-t$ Min CUT, which becomes strongly $N P$-hard when considering its min-max or minmax regret version.

Min-max cut was proved polynomially solvable for a constant number of scenarios [3]. We show that min-max regret cut also remains polynomial for a constant number of scenarios. When the number of scenarios is not constant, min-max (regret) versions become strongly $N P$-hard.

\section{$3.1 s-t$ min cut}

In order to prove these results, we construct polynomial reductions from the decision version of Min Bisection.

Theorem 1. Discrete Min-Max (Regret) $s-t$ Cut are strongly NP-hard even for two scenarios.

Proof. Consider an instance $G=(V, E)$ of Min Bisection with $|V|=2 n$, and a positive integer $v$. We construct an instance $\widetilde{G}=(\widetilde{V}, \widetilde{E})$ of Discrete Min-Max $s-t$ CUT with the scenario set $S=\left\{s_{1}, s_{2}\right\}$. The node set is $\widetilde{V}=V \cup\{s, t\}$ where $s$ and $t$ correspond to a source and a sink respectively. The edge set $\widetilde{E}=E \cup\{(s, i): i \in V\} \cup\{(i, t): i \in V\}$. Edge weights in scenarios $s_{1}$ and $s_{2}$ are assigned for each edge $(i, j) \in \widetilde{E}$ as follows:

$$
w_{i j}^{1}=\left\{\begin{array}{ll}
1 & \text { if }(i, j) \in E \\
n^{2}+1 & \text { if } i=s \text { or } j=s \\
0 & \text { if } i=t \text { or } j=t
\end{array} \quad \text { and } \quad w_{i j}^{2}= \begin{cases}1 & \text { if }(i, j) \in E \\
0 & \text { if } i=s \text { or } j=s \\
n^{2}+1 & \text { if } i=t \text { or } j=t\end{cases}\right.
$$

We claim that there exists a bisection $C$ in $G$ of value at most $v$ if and only if there exists an $s-t$ cut $\widetilde{C}$ in $\widetilde{G}$ with $\max \left\{\operatorname{val}\left(\widetilde{C}, s_{1}\right), \operatorname{val}\left(\widetilde{C}, s_{2}\right)\right\} \leq v+\left(n^{2}+1\right) n$. $\Rightarrow$ Consider a bisection $C=\left(V_{1}, V_{2}\right)$ of value $x \leq v$. We construct an $s-t$ cut $\widetilde{C}=\left(\widetilde{V}_{1}, \widetilde{V}_{2}\right)$ where $\widetilde{V}_{1}=V_{1} \cup\{s\}$, and $\widetilde{V}_{2}=V_{2} \cup\{t\}$. Consequently, we have $\operatorname{val}\left(\widetilde{C}, s_{1}\right)=\operatorname{val}\left(\widetilde{C}, s_{2}\right)=x+\left(n^{2}+1\right) n \leq v+\left(n^{2}+1\right) n$. 
$\Leftarrow$ Consider now an $s-t$ cut $\widetilde{C}=\left(\widetilde{V}_{1}, \widetilde{V}_{2}\right)$ verifying $\max \left\{\operatorname{val}\left(\widetilde{C}, s_{1}\right), \operatorname{val}\left(\widetilde{C}, s_{2}\right)\right\} \leq$ $v+\left(n^{2}+1\right) n$. Let $V_{1}=\widetilde{V}_{1} \backslash\{s\}$ and $V_{2}=\widetilde{V}_{2} \backslash\{t\}$. We have by construction $\operatorname{val}\left(\widetilde{C}, s_{1}\right)=y+\left|V_{2}\right|\left(n^{2}+1\right)$ and $\operatorname{val}\left(\widetilde{C}, s_{2}\right)=y+\left|V_{1}\right|\left(n^{2}+1\right)$, where $y$ is the number of edges from $E$ that have one endpoint in $V_{1}$ and one endpoint in $V_{2}$. Suppose that $\left|V_{1}\right|=n+z$ and $\left|V_{2}\right|=n-z, z \geq 0$. Then $\operatorname{val}\left(\widetilde{C}, s_{1}\right)=y+\left(n^{2}+1\right) n-$ $z\left(n^{2}+1\right), \operatorname{val}\left(\widetilde{C}, s_{2}\right)=y+\left(n^{2}+1\right) n+z\left(n^{2}+1\right)$ and $\max \left\{\operatorname{val}\left(\widetilde{C}, s_{1}\right), \operatorname{val}\left(\widetilde{C}, s_{2}\right)\right\}=$ $y+\left(n^{2}+1\right) n+z\left(n^{2}+1\right) \leq v+\left(n^{2}+1\right) n$. Since $v \leq n^{2}$ we have $z=0$ and thus $\left|V_{1}\right|=\left|V_{2}\right|=n$ and $y \leq v$.

In order to prove the result for the min-max regret version, we use exactly the same graph $\widetilde{G}=(\widetilde{V}, \widetilde{E})$. Let $C_{i}^{*}$ denote the optimal solution in scenario $s_{i}$, $i=1,2$. We have $C_{1}^{*}=(\widetilde{V} \backslash\{t\},\{t\})$ and $C_{2}^{*}=(\{s\}, \widetilde{V} \backslash\{s\})$ with $\operatorname{val}\left(C_{1}^{*}, s_{1}\right)=$ $\operatorname{val}\left(C_{2}^{*}, s_{2}\right)=0$. Therefore, there exists a bisection in $G$ of value at most $v$ if and only if there exists an $s-t$ cut $\widetilde{C}$ in $\widetilde{G}$ with $R_{\max }(\widetilde{C}) \leq v+\left(n^{2}+1\right) n$.

\section{$3.2 \quad$ Min cut}

Armon and Zwick [3] constructed a polynomial-time algorithm for DiscReTE Min-MaX Cut, in the case of a constant number of scenarios, based essentially on the result of Nagamochi, Nishimura and Ibaraki [8] for computing all $\alpha$ approximate cuts in time $O\left(m^{2} n+m n^{2 \alpha}\right)$. A cut $C$ in a graph $G$ is called an $\alpha$-approximate cut if $\operatorname{val}(C) \leq \alpha$ opt, where opt is the value of a minimum cut in $G$.

Theorem 2 ([3]). Discrete Min-Max CUT is solvable in polynomial time for a constant number of scenarios.

In a graph on $n$ vertices and $m$ edges and with $k$ scenarios, Armon and Zwick's algorithm [3] constructs an optimal solution in $O\left(m n^{2 k}\right)$.

We show in the following that this algorithm can be modified in order to obtain a polynomial-time algorithm for Discrete Min-Max Regret Cut.

Theorem 3. Discrete Min-Max Regret Cut is solvable in polynomial time for a constant number of scenarios.

Proof. Consider an instance $I$ of the problem given by graph $G=(V, E)$ on $n$ vertices and $m$ edges and a set of $k$ scenarios $S$ such that each edge $(i, j) \in E$ has a weight $w_{i j}^{s}$ in scenario $s$. We construct, as before, an instance $I^{\prime}$ of MiN CuT on the same graph, where $w_{i j}^{\prime}=\sum_{s \in S} w_{i j}^{s}$. The algorithm consists firstly of computing all $k$-approximate cuts and secondly of choosing among these cuts one with a minimum maximum regret.

The running time of the algorithm is $O\left(m n^{2 k}\right)$.

We prove now the correctness of the algorithm. Let $C^{*}$ be an optimal minmax regret cut in $G$. We show that for any cut $C$ of $G$, we have $\operatorname{val}^{\prime}\left(C^{*}\right) \leq$ $\operatorname{kval}^{\prime}(C)$, where $\operatorname{val}^{\prime}(C)$ is the value of cut $C$ in $I^{\prime}$. In fact,

$$
\operatorname{val}^{\prime}\left(C^{*}\right)=\sum_{s \in S} \operatorname{val}\left(C^{*}, s\right)=\sum_{s \in S}\left(\operatorname{val}\left(C^{*}, s\right)-\operatorname{val}_{s}^{*}\right)+\sum_{s \in S} \operatorname{val}_{s}^{*} \leq
$$




$$
\begin{aligned}
& \left.k \max _{s \in S}\left\{\operatorname{val}\left(C^{*}, s\right)-\operatorname{val}_{s}^{*}\right\}+\sum_{s \in S} \operatorname{val}_{s}^{*} \leq k \max _{s \in S}\left\{\operatorname{val}(C, s)-\operatorname{val}_{s}^{*}\right)\right\}+\sum_{s \in S} \operatorname{val}_{s}^{*} \leq \\
& k \sum_{s \in S}\left(\operatorname{val}(C, s)-\operatorname{val}_{s}^{*}\right)+\sum_{s \in S} \operatorname{val}_{s}^{*}=k \sum_{s \in S} \operatorname{val}(C, s)-(k-1) \sum_{s \in S} \operatorname{val}_{s}^{*} \leq \operatorname{kval}^{\prime}(C)
\end{aligned}
$$

In particular, if $C$ is a minimum cut in $I^{\prime}$, we obtain $\operatorname{val}^{\prime}\left(C^{*}\right) \leq k o p t\left(I^{\prime}\right)$. Thus all optimal solutions to Discrete Min-Max Regret Cut are among the $k$-approximate cuts in $I^{\prime}$.

The algorithms described above to solve Discrete Min-Max (Regret) CuT are exponential in $k$. We prove in the following that when $k$ is not constant, both problems become strongly $N P$-hard.

Theorem 4. Discrete Min-Max (Regret) Cut are strongly NP-hard for a non constant number of scenarios.

Proof. We use a reduction from Min Bisection. Consider an instance $G=$ $(V, E)$ of Min Bisection with $V=\{1, \ldots, 2 n\}$, and a positive integer $v$. We construct an instance $\widetilde{G}=(\widetilde{V}, \widetilde{E})$ of Discrete Min-Max CUT with a scenario set $S$ of size $2 n$. The node set is $\widetilde{V}=V \cup\left\{1^{\prime}, \ldots, 2 n^{\prime}\right\} \cup\left\{1^{\prime \prime}, \ldots, 2 n^{\prime \prime}\right\}$. The edge set $\widetilde{E}=E \cup\left\{\left(i^{\prime}, j^{\prime}\right): i, j=1, \ldots, 2 n\right\} \cup\left\{\left(i, i^{\prime}\right),\left(i^{\prime}, i^{\prime \prime}\right): i=1, \ldots, 2 n\right\}$. Scenario set $S$ corresponds to nodes of $G$. The weights of the edges in any scenario $s_{i} \in S$ are defined as follows: $w_{h j}^{i}=1$ for all $(h, j) \in E ; w_{i^{\prime} j^{\prime}}^{i}=n^{2}$, for $j=1, \ldots, 2 n$; $w_{h^{\prime} j^{\prime}}^{i}=0$ for $h \neq i$ and $j \neq i ; w_{i i^{\prime}}^{i}=w_{i^{\prime} i^{\prime \prime}}^{i}=n^{3}+n^{2}+1 ; w_{j j^{\prime}}^{i}=w_{j^{\prime} j^{\prime \prime}}^{i}=0$, for $j \neq i$.

We claim that there exists a bisection $C$ in $G$ of value at most $v$ if and only if there is a cut $\widetilde{C}$ in $\widetilde{G}$ with $\max _{s \in S} \operatorname{val}(\widetilde{C}, s) \leq n^{3}+v$.

$\Rightarrow$ Consider a bisection $C=\left(V_{1}, V_{2}\right)$ in $G$ of value $x \leq v$. We construct a cut $\widetilde{C}=\left(\widetilde{V}_{1}, \widetilde{V}_{2}\right)$ in $\widetilde{G}$ where $\widetilde{V}_{1}=V_{1} \cup\left\{i^{\prime}, i^{\prime \prime}: i \in V_{1}\right\}$ and $\widetilde{\widetilde{V}}_{2}=V_{2} \cup\left\{i^{\prime}, i^{\prime \prime}: i \in V_{2}\right\}$. For any scenario $s \in S$, we have $\operatorname{val}(\widetilde{C}, s) \leq n^{3}+v$ and thus $\max _{s \in S} \operatorname{val}(\widetilde{C}, s) \leq$ $n^{3}+v$.

$\Leftarrow$ Consider now a cut $\widetilde{C}=\left(\widetilde{V}_{1}, \widetilde{V}_{2}\right)$ in $\widetilde{G}$ such that $\max _{s \in S} \operatorname{val}(\widetilde{C}, s) \leq n^{3}+v$. Cut $\widetilde{C}$ does not contain any edge $\left(i, i^{\prime}\right)$ or $\left(i^{\prime}, i^{\prime \prime}\right)$ for some $i=1, \ldots, 2 n$, since otherwise, we have $\max _{s \in S} \operatorname{val}(\widetilde{C}, s) \geq n^{3}+n^{2}+1>n^{3}+v$. Denote by $V_{i}$, for $i=1,2$, the restriction of $\widetilde{V}_{i}$ to the vertices of $V$. Suppose now that $\left|V_{1}\right|<\left|V_{2}\right|$, then for any scenario $s_{i}$ such that $i \in V_{1}$ we have $\operatorname{val}\left(\widetilde{C}, s_{i}\right) \geq(n+1) n^{2}+1>$ $n^{3}+v$. Thus, we have necessarily $\left|V_{1}\right|=\left|V_{2}\right|$ and the value of the bisection $\left(V_{1}, V_{2}\right)$ is at most $v$.

In order to prove the result for the min-max regret version, we use exactly the same graph $\widetilde{G}=(\widetilde{V}, \widetilde{E})$. Notice that, for any scenario $s_{i} \in S$, cut $C_{i}^{*}=$ $\left(\left\{j^{\prime \prime}\right\}, \widetilde{V} \backslash\left\{j^{\prime \prime}\right\}\right)$ for some $j \neq i$ is a minimum cut in scenario $s_{i}$, with value 0 . Therefore, there exists a bisection in $G$ of value at most $v$ if and only if there exists a cut $\widetilde{C}$ in $\widetilde{G}$ with $R_{\max }(\widetilde{C}) \leq n^{3}+v$.

Observe that in the previous proof we used the same graph $\widetilde{G}$ both for the min-max and min-max regret versions. Actually, a slightly simpler proof can be 
obtained, for the min-max part, considering only the subgraph of $\widetilde{G}$ induced by $\widetilde{V} \backslash\left\{1^{\prime \prime}, \ldots, 2 n^{\prime \prime}\right\}$. Vertex subset $\left\{1^{\prime \prime}, \ldots, 2 n^{\prime \prime}\right\}$ is necessary, for the min-max regret part, to ensure the existence of minimum cuts of value 0 for each scenario.

\section{Interval data case}

We first state the polynomiality of the min-max cut problems (Section 4.1), then we establish the strong $N P$-hardness of Interval Min-Max REGReT $s-t$ Cut (Section 4.2) and the polynomiality of Interval Min-Max Regret Cut (Section 4.2).

\subsection{Min-max versions}

In the interval data case, the min-max version of a minimization problem corresponds to solving this problem in the worst-case scenario defined by the upper bounds of all intervals. Therefore, a minimization problem and its min-max version have the same complexity. Interval Min-Max $s-t$ Cut and Interval Min-Max Cut are thus polynomial-time solvable.

\subsection{Min-max regret versions}

When the number $u \leq m$ of uncertain/imprecise parameters, corresponding to non-degenerate intervals, is small enough, then the problem becomes polynomial. More precisely, as shown by Averbakh and Lebedev [5] for general networks problems solvable in polynomial time, if $u$ is fixed or bounded by the logarithm of a polynomial function of $m$, then the min-max regret version is also solvable in polynomial time (based on the fact that an optimal solution for the min-max regret version corresponds to one of the optimal solutions for the $2^{u}$ extreme scenarios, where extreme scenarios have values on each edge corresponding to either the lower or upper bound of its interval). This clearly applies to the $s-t$ min cut and min cut problems.

\section{$s-t$ min cut}

We show now that Interval Min-Max Regret $s-t$ Cut is strongly $N P$ hard. For this purpose, we construct a reduction from the decision version of $s-t$ Min BiseCtion.

Theorem 5. Interval Min-Max Regret $s-t$ CUt is strongly NP-hard.

Proof. Consider $G=(V, E)$ an instance of $s-t$ Min Bisection with $|V|=2 n$, where $V=\{s=1, \ldots, t=2 n\}$. We construct from $G$ an instance $\widetilde{G}=(\widetilde{V}, \widetilde{E})$ of Interval Min-Max Regret $s-t$ CUt as illustrated in Figure 1 . The vertex set is $\widetilde{V}=V \cup\left\{1^{\prime}, \ldots, 2 n^{\prime}\right\} \cup\left\{1^{\prime \prime}, \ldots, 2 n^{\prime \prime}\right\} \cup\left\{1^{\prime \prime \prime}, \ldots, 2 n^{\prime \prime \prime}\right\} \cup\{\widetilde{s}, 2 n+1\}$, and $\widetilde{t}=t$. 
The edge set is $\widetilde{E}=E \cup\left\{\left(i^{\prime}, i^{\prime \prime}\right),\left(i^{\prime \prime}, i^{\prime \prime \prime}\right): i=1, \ldots, 2 n\right\} \cup\left\{\left(i, i^{\prime \prime}\right): i=\right.$ $2, \ldots, 2 n-1\} \cup\left\{\left(2 n+1, i^{\prime}\right): i=1, \ldots, 2 n\right\} \cup\left\{\left(i^{\prime \prime \prime}, t\right): i=1, \ldots, 2 n\right\} \cup$ $\{(\widetilde{s}, 2 n+1),(\widetilde{s}, s)\}$.

Let $p$ and $q$ verifying, respectively, $p>n^{2}$ and $q>4 n(p+1)^{2}$. The weights are defined as follows :

$$
\begin{aligned}
& -\underline{w}_{i j}=\bar{w}_{i j}=1 \text {, for all }(i, j) \in E \text {; } \\
& -\underline{w}_{i^{\prime} i^{\prime \prime}}=\left\{\begin{array}{l}
q \text { for } i=1 \\
0 \text { otherwise }
\end{array} \text { and } \bar{w}_{i^{\prime} i^{\prime \prime}}=\left\{\begin{array}{l}
q \quad \text { for } i=1 \\
p^{2}+p \text { otherwise }
\end{array}\right.\right. \\
& -\underline{w}_{i^{\prime \prime} i^{\prime \prime \prime}}=\bar{w}_{i^{\prime \prime} i^{\prime \prime \prime}}= \begin{cases}p^{2}+n p & \text { for } i=1 \\
p^{2} & \text { for } i=2, \ldots, 2 n-1 \\
q & \text { for } i=2 n\end{cases} \\
& -\underline{w}_{i i^{\prime \prime}}=\bar{w}_{i i^{\prime \prime}}=q \text {, for } i=2, \ldots, 2 n-1 \text {; } \\
& -\underline{w}_{(2 n+1) i^{\prime}}=\left\{\begin{array}{l}
0 \text { for } i=1 \\
2 p \text { otherwise }
\end{array} \text { and } \bar{w}_{(2 n+1) i^{\prime}}=q, \text { for } i=1, \ldots, 2 n\right. \text {; } \\
& -\underline{w}_{i^{\prime \prime \prime} t}=\bar{w}_{i^{\prime \prime \prime} t}=q \text {, for } i=1, \ldots, 2 n \text {; } \\
& -\underline{w}_{\widetilde{s}(2 n+1)}=2 n p \text { and } \bar{w}_{\widetilde{s}(2 n+1)}=q \text {; } \\
& -\underline{w}_{\widetilde{s} s}=0 \text { and } \bar{w}_{\widetilde{s} s}=q \text {. }
\end{aligned}
$$

Clearly this transformation can be obtained in polynomial time.

We first establish the following property.

For any $\widetilde{s}-\widetilde{t}$ cut $\widetilde{C}=\left(\widetilde{V}_{1}, \widetilde{V}_{2}\right)$ in $\widetilde{G}$ not including any edge $(i, j) \in \widetilde{E}$ with $\bar{w}_{i j}=q$, a minimum $\widetilde{s}-\widetilde{t}$ cut $C_{w^{-}(\widetilde{C})}^{*}$ in, $w^{-}(\widetilde{C})$, the worst scenario associated to $\widetilde{C}$, has value $\operatorname{val}\left(C_{w^{-}(\widetilde{C})}^{*}, w^{-}(\widetilde{C})\right)=2 p \min \left\{n,\left|V_{2}\right|\right\}$, where $V_{2}=\widetilde{V}_{2} \cap V$.

Indeed, consider such a cut $\widetilde{C}=\left(\widetilde{V}_{1}, \widetilde{V}_{2}\right)$ with $\widetilde{s} \in \widetilde{V}_{1}, \widetilde{t} \in \widetilde{V}_{2}$ and denote $V_{1}=\widetilde{V}_{1} \cap V$. Clearly, vertices $2 n+1,1^{\prime \prime}$ and $i^{\prime}, i=1, \ldots, 2 n$ belong to $\widetilde{V}_{1}$. Also, vertices $2 n^{\prime \prime}$ and $i^{\prime \prime \prime}, i=1, \ldots, 2 n$ belong to $\widetilde{V}_{2}$. Moreover, $i$ and $i^{\prime \prime}$ belong to the same part, $\widetilde{V}_{1}$ or $\widetilde{V}_{2}$. It follows that

$$
\operatorname{val}\left(\widetilde{C}, w^{-}(\widetilde{C})\right)=x+\left(n+\left|V_{2}\right|\right) p+2 n p^{2}
$$

where $x$ denotes the number of edges that have one endpoint in $V_{1}$ and one endpoint in $V_{2}$.

By construction, $C_{w^{-}(\widetilde{C})}^{*}$ necessarily cuts edge $(\widetilde{s}, s)$. Furthermore, there exist two cases:

1. If $\left|V_{2}\right| \leq n$ then $C_{w^{-}(\widetilde{C})}^{*}=\left(\widetilde{V}_{1}^{*}, \widetilde{V}^{*} \backslash \widetilde{V}_{1}^{*}\right)$, where $\widetilde{V}_{1}^{*}=\{\widetilde{s}, 2 n+1\} \cup\left\{i^{\prime}: i^{\prime \prime} \in\right.$ $\left.\widetilde{V}_{1}, i \neq 1\right\}$ and thus $\operatorname{val}\left(C_{w^{-}(\widetilde{C})}^{*}, w^{-}(\widetilde{C})\right)=2\left|V_{2}\right| p$.

2. If $\left|V_{2}\right|>n$ then $C_{w^{-}(\widetilde{C})}^{*}=(\{\widetilde{s}\}, \widetilde{V} \backslash\{\widetilde{s}\})$ and thus $\operatorname{val}\left(C_{w^{-}(\widetilde{C})}^{*}, w^{-}(\widetilde{C})\right)=2 n p$. 


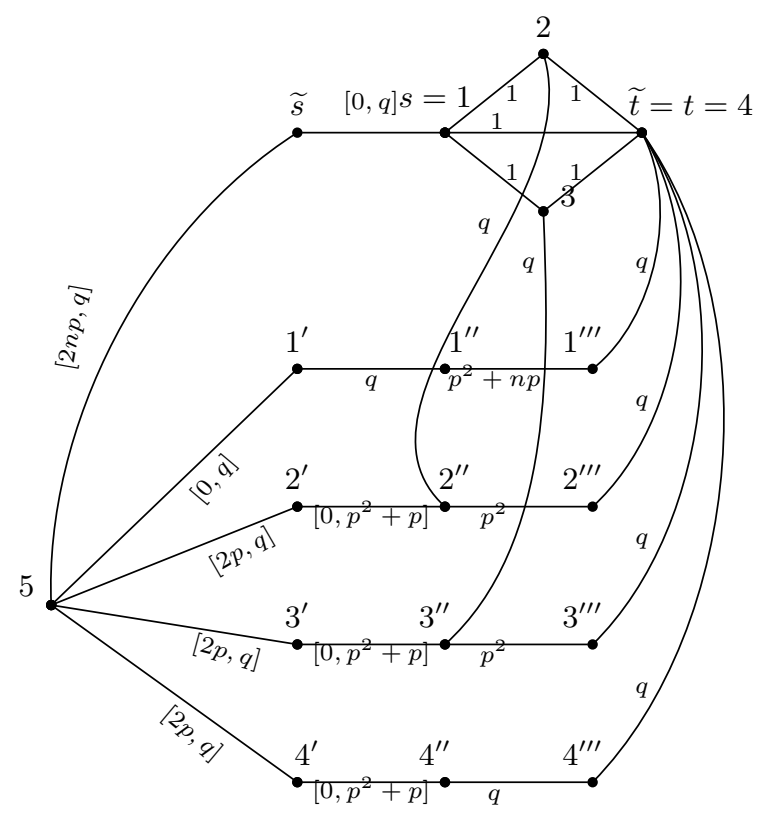

Fig. 1. Interval Min-Max Regret $s-t$ Cut instance resulting from $s-t$ Min Bisection instance.

We claim that there exists an $s-t$ bisection $C=\left(V_{1}, V_{2}\right)$ of value no more than $v$ if and only if there exists an $\widetilde{s}-\widetilde{t}$ cut $\widetilde{C}=\left(\widetilde{V}_{1}, \widetilde{V}_{2}\right)$ in $\widetilde{G}$ with $R_{\max }(\widetilde{C}) \leq$ $v+2 n p^{2}$.

$\Rightarrow$ Consider an $s-t$ bisection $C=\left(V_{1}, V_{2}\right)$ in $G$ of value $x \leq v$. We construct an $\widetilde{s}-\widetilde{t}$ cut $\widetilde{C}$ in $\widetilde{G}$ deduced from $C$ as follows: $\widetilde{V}_{1}=\{\widetilde{s}, 2 n+1\} \cup\left\{1^{\prime}, \ldots, 2 n^{\prime}\right\} \cup$ $V_{1} \cup\left\{i^{\prime \prime}: i \in V_{1}\right\}$ and $\widetilde{V}_{2}=\left\{1^{\prime \prime \prime}, \ldots, 2 n^{\prime \prime \prime}\right\} \cup V_{2} \cup\left\{i^{\prime \prime}: i \in V_{2}\right\}$. It is easy to verify that $\operatorname{val}\left(\widetilde{C}, w^{-}(\widetilde{C})\right)=x+2 n\left(p+p^{2}\right)$ and using the previous result, we have $R_{\text {max }}(\widetilde{C})=x+2 n p^{2} \leq v+2 n p^{2}$.

$\Leftarrow$ Consider an $\widetilde{s}-\widetilde{t}$ cut $\widetilde{C}$ in $\widetilde{G}$ with $R_{\max }(\widetilde{C}) \leq v+2 n p^{2}$. Cut $\widetilde{C}$ does not cut any edge $(i, j) \in \widetilde{E}$ such that $\bar{w}_{i j}=q$, since otherwise, $\operatorname{val}\left(\widetilde{C}, w^{-}(\widetilde{C})\right) \geq q$, and, since a minimum $\widetilde{s}-\widetilde{t}$ cut $C_{w^{-}(\widetilde{C})}^{*}$ in $w^{-}(\widetilde{C})$, does not cut any edge $(i, j) \in \widetilde{E}$ such that $\bar{w}_{i j}=q$, we have, using $(1), \operatorname{val}\left(C_{w^{-}(\widetilde{C})}^{*}, w^{-}(\widetilde{C})\right) \leq n^{2}+3 n p+2 n p^{2}<4 n p+2 n p^{2}$ and consequently, we have $R_{\max }(\widetilde{C})>2 n p^{2}+v$.

Thus $\operatorname{val}\left(\widetilde{C}, w^{-}(\widetilde{C})\right)=y+2 n p^{2}+n p+p\left|V_{2}\right|$ where $y$ is the value of the cut induced by $\widetilde{C}$ in $E$. It follows that

$$
R_{\max }(\widetilde{C})=\left\{\begin{array}{l}
y+\left(n-\left|V_{2}\right|\right) p+2 n p^{2} \text { if }\left|V_{2}\right| \leq n \\
y+\left(\left|V_{2}\right|-n\right) p+2 n p^{2} \text { if }\left|V_{2}\right|>n
\end{array}\right.
$$


Consequently, since $R_{\max }(\widetilde{C}) \leq v+2 n p^{2}$, and $p>n^{2} \geq v$, we have $\left|V_{1}\right|=n=$ $\left|V_{2}\right|$ and $y \leq v$.

\section{Min cut}

We prove in this section that the min-max regret version of min-cut problem is polynomial in the interval data case.

Theorem 6. Interval Min-Max Regret Cut is solvable in polynomial time in the interval data case.

Proof. Consider an instance $I$ of Interval Min-Max Regret Cut given by graph $G=(V, E)$ on $n$ vertices and $m$ edges. The weight $w_{i j}$ of each edge $(i, j) \in E$ can take any value in the interval $\left[\underline{w}_{i j}, \bar{w}_{i j}\right]$. We construct an instance $I^{\prime}$ of Min CUT on the same graph, where $w_{i j}^{\prime}=\bar{w}_{i j}$. The algorithm consists firstly of computing all the 2-approximate minimum cuts in $I^{\prime}$ and secondly of choosing among these cuts one with a minimum maximum regret.

The running time of the algorithm is $O\left(m n^{5}+n^{6} \log m\right)$.

We prove now the correctness of the algorithm. Let $C^{*}$ be an optimal cut in $I$ and $v a l^{\prime}(C)$ denote the value of any cut $C$ in $I^{\prime}$. Then the following inequalities hold:

$$
\begin{aligned}
\operatorname{val}^{\prime}\left(C^{*}\right) & =R_{\max }\left(C^{*}\right)+\operatorname{val}_{w^{-}}^{*}\left(C^{*}\right) \\
& \leq R_{\max }(C)+\operatorname{val}\left(C, w^{-}\left(C^{*}\right)\right) \leq 2 \operatorname{val}^{\prime}(C)
\end{aligned}
$$

In particular, if $C$ is a minimum cut in $I^{\prime}$, we obtain $v a l^{\prime}\left(C^{*}\right) \leq 2 o p t\left(I^{\prime}\right)$. Thus all optimal solutions to Interval Min-Max Regret Cut are among the 2approximate cuts in $I^{\prime}$.

\section{References}

1. H. Aissi, C. Bazgan, and D. Vanderpooten. Approximation complexity of min-max (regret) versions of shortest path, spanning tree, and knapsack. In Proceedings of the 13th Annual European Symposium on Algorithms (ESA 2005), Mallorca, Spain, 2005. to appear.

2. H. Aissi, C. Bazgan, and D. Vanderpooten. Complexity of the min-max and minmax regret assignment problem. Operations Research Letters, 2005. to appear.

3. A. Armon and U. Zwick. Multicriteria global minimum cuts. In Proceedings of the 15th International Symposium on Algorithms and Complexity (ISAAC 2004), Hong Kong, China, LNCS 3341, pages 65-76. Springer-Verlag, 2004.

4. I. D. Aron and P. Van Hentenryck. On the complexity of the robust spanning tree with interval data. Operations Research Letters, 32:36-40, 2004.

5. I. Averbakh and V. Lebedev. Interval data min-max regret network optimization problems. Discrete Applied Mathematics, 138:289-301, 2004.

6. M. Garey and D. Johnson. Computers and Intractability: A Guide to the theory of NP-completeness. San Francisco, 1979.

7. P. Kouvelis and G. Yu. Robust Discrete Optimization and its Applications. Kluwer Academic Publishers, Boston, 1997. 
8. H. Nagamochi, K. Nishimura, and T. Ibaraki. Computing all small cuts in an undirected network. SIAM Journal on Discrete Mathematics, 10(3):469-481, 1997.

9. H. Yaman, O. E. Karaşan, and M. C. Pinar. The robust spanning tree problem with interval data. Operations Research Letters, 29:31-40, 2001. 